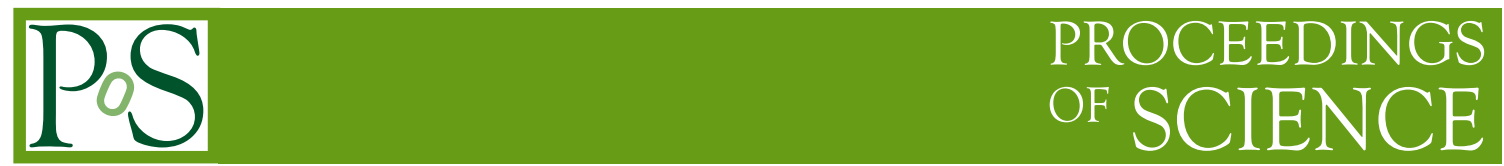

\title{
Can complex Langevin dynamics evade the sign problem?
}

\section{Gert Aarts*}

Physics Department, Swansea University, Swansea, United Kingdom

E-mail: g.aarts@swan.ac.uk

I answer the question in the title for the relativistic Bose gas at finite chemical potential using numerical lattice simulations, complemented with analytical understanding.

The XXVII International Symposium on Lattice Field Theory

July 26-31, 2009

Peking University, Beijing, China

*Speaker. 


\section{Introduction}

As is well known, at nonzero baryon chemical potential the complexity of the fermion determinant prohibits the use of importance sampling in lattice QCD. This makes the determination of the QCD phase diagram an outstanding open problem (a summary of what is possible despite this obstruction can be found in Ref. [1]). Stochastic quantization [2] does not rely on importance sampling: the configurations that dominate in the partition function are found by integrating complex Langevin equations in an enlarged phase space, after a complexification of the degrees of freedom $[3,4,5]$. During last year's Conference I reported [6] on work done in collaboration with IonOlimpiu Stamatescu on QCD with static quarks and related one-link models [7]. In the one-link models we found excellent results for all values of the chemical potential, from zero all the way to saturation. Moreover, the sign problem was present but clearly not dangerous. The results in the heavy dense limit of QCD were preliminary but encouraging.

These results triggered a number of intriguing questions. For instance, can complex Langevin dynamics handle

- a severe sign problem?

- the thermodynamic limit?

- phase transitions?

- the Silver Blaze problem [8]?

I will answer these questions positively in a theory that is much simpler than QCD: the relativistic Bose gas at nonzero chemical potential [9]. Even though it lacks the intricacies of a nonabelian gauge theory, it is a four-dimensional lattice field theory with a sign problem, a Silver Blaze problem, and a phase transition. As an additional benefit, one can study the complex Langevin equations perturbatively, allowing detailed analytical insight into the algorithm [10]. Of course, there are many more questions to ask and work to address those is currently in progress.

\section{Relativistic Bose gas}

The lattice action is

$$
S=\sum_{x}\left[\left(2 d+m^{2}\right) \phi_{x}^{*} \phi_{x}+\lambda\left(\phi_{x}^{*} \phi_{x}\right)^{2}-\sum_{v=1}^{4}\left(\phi_{x}^{*} e^{-\mu \delta_{v, 4}} \phi_{x+\hat{v}}+\phi_{x+\hat{v}}^{*} e^{\mu \delta_{v, 4}} \phi_{x}\right)\right]
$$

where $d=4$ and we take $m^{2}>0$. It satisfies $S^{*}(\mu)=S\left(-\mu^{*}\right)$, just as in QCD. In continuum notation, the nonderivative part of the Lagrangian density reads $\left(m^{2}-\mu^{2}\right)|\phi|^{2}+\lambda|\phi|^{4}$ : at tree level, a phase transition at $\mu_{c}=m$ separates the vacuum (symmetric) and the high-density (symmetrybroken) phases. The $\mu$-independence of bulk thermodynamic quantities as long as $\mu<\mu_{c}$, even though the action, the Boltzmann weight, and hence the field configurations depend explicitly on $\mu$, is the Silver Blaze problem [8]. Equating the imaginary part of the action with zero yields the phase quenched theory: this is a theory with a $\mu$-dependent mass parameter and no sign problem. 
After decomposing $\phi=\left(\phi_{1}+i \phi_{2}\right) / \sqrt{2}$ and complexifying $\phi_{a} \rightarrow \phi_{a}^{\mathrm{R}}+\phi_{a}^{\mathrm{I}}(a=1,2)$, it is straightforward to write down the complex Langevin equations for the (now) four real fields,

$$
\frac{\partial \phi_{a}^{\mathrm{R}}}{\partial \theta}=-\left.\operatorname{Re} \frac{\delta S}{\delta \phi_{a}}\right|_{\phi_{a} \rightarrow \phi_{a}^{\mathrm{R}}+i \phi_{a}^{\mathrm{I}}}+\eta_{a}, \quad \frac{\partial \phi_{a}^{\mathrm{I}}}{\partial \theta}=-\left.\operatorname{Im} \frac{\delta S}{\delta \phi_{a}}\right|_{\phi_{a} \rightarrow \phi_{a}^{\mathrm{R}}+i \phi^{\mathrm{I}}},
$$

where $\theta$ is the Langevin time and $\eta_{a}$ is Gaussian noise with $\left\langle\eta_{a} \eta_{b}\right\rangle=2 \delta_{a b}$. It is also straightforward to solve these equations numerically [9]. I have used $m=\lambda=1$ on lattices of size $\Omega=N^{4}$, with $N=4,6,8,10$, and Langevin time step $\varepsilon=5 \times 10^{-5}$ and not encountered instabilities in the numerical integration. The phase quenched theory is solved using real Langevin dynamics (with $\left.\phi^{\mathrm{I}} \equiv 0\right)$.

The resulting density $\langle n\rangle=\Omega^{-1} \partial \ln Z / \partial \mu$ is shown in Fig. 1, in the full theory (left) and the phase quenched theory (right). The Silver Blaze feature in the full theory is immediately visible. In the thermodynamic limit the density is strictly zero as long as $\mu<\mu_{c} \approx 1.15$. The phase quenched theory has no Silver Blaze region: instead the density increases as soon as $\mu$ is nonzero. The $\mu$-independence in the full theory is a direct result of the complexity of the action and the sign problem.

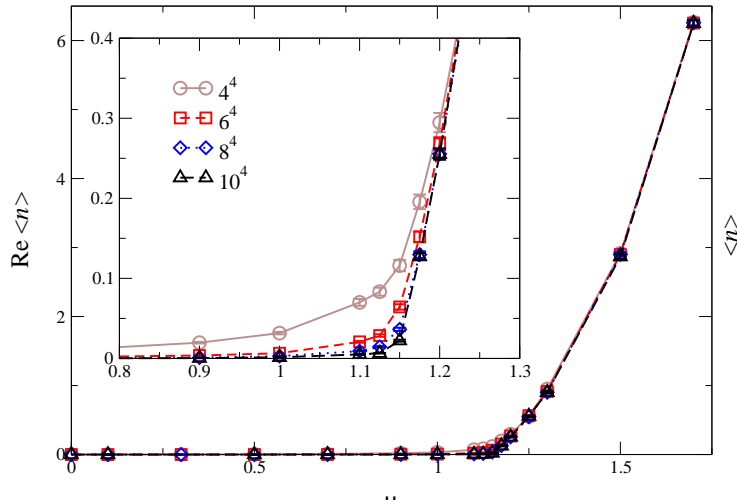

$\mu$

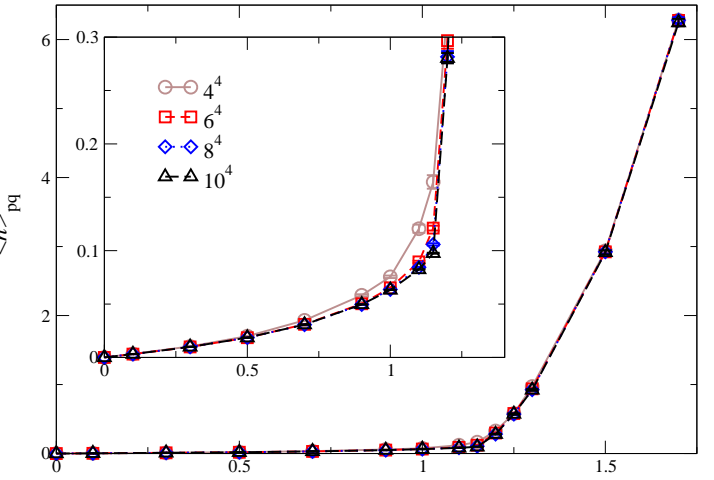

$\mu$

Figure 1: Density in the full (left) and the phase quenched (right) theory as a function of chemical potential on lattices with size $N^{4}$, with $N=4,6,8,10(m=\lambda=1)$.

In this model the complex Langevin equations can be studied perturbatively [10]. Ignoring interactions, the solution of these equations reads, in momentum space and in the Silver Blaze region only,

$$
\begin{aligned}
\phi_{a, p}^{\mathrm{R}}(\theta)= & e^{-A_{p} \theta}\left[\cos \left(B_{p} \theta\right) \phi_{a, p}^{\mathrm{R}}(0)+i \sin \left(B_{p} \theta\right) \varepsilon_{a b} \phi_{b, p}^{\mathrm{I}}(0)\right] \\
& +\int_{0}^{\theta} d s e^{-A_{p}(\theta-s)} \cos \left[B_{p}(\theta-s)\right] \eta_{a, p}(s), \\
\phi_{a, p}^{\mathrm{I}}(\theta)= & e^{-A_{p} \theta}\left[\cos \left(B_{p} \theta\right) \phi_{a, p}^{\mathrm{I}}(0)-i \sin \left(B_{p} \theta\right) \varepsilon_{a b} \phi_{b, p}^{\mathrm{R}}(0)\right] \\
& -i \int_{0}^{\theta} d s e^{-A_{p}(\theta-s)} \sin \left[B_{p}(\theta-s)\right] \varepsilon_{a b} \eta_{b, p}(s) .
\end{aligned}
$$


Here $\phi_{a, p}^{\mathrm{R}, \mathrm{I}}(0)$ are the initial conditions, $\varepsilon_{a b}$ is the totally antisymmetric tensor with $\varepsilon_{11}=1$, and the coefficients $A_{p}, B_{p}$ are

$$
\begin{aligned}
A_{p} & =m^{2}+4 \sum_{i=1}^{3} \sin ^{2} \frac{p_{i}}{2}+2\left(1-\cosh \mu \cos p_{4}\right) \rightarrow m^{2}-\mu^{2}+\mathbf{p}^{2}+p_{4}^{2}, \\
B_{p} & =2 \sinh \mu \sin p_{4} \rightarrow 2 \mu p_{4} .
\end{aligned}
$$

The expressions after the arrows correspond to the formal continuum limit. This solution is only valid when $A_{p}>0$ (or $\mu<\mu_{c}$ ), which reflects the standard instability of the free Bose gas. When $\mu>\mu_{c}$, symmetry breaks and the inclusion of the $\lambda|\phi|^{4}$ term is necessary for stabilization.

From the analytical solution, we find that both the independence of initial conditions and convergence at large Langevin time are controlled by $e^{-A_{p} \theta}$. This is demonstrated numerically for the interacting theory in Fig. 2 for $\mu=0.5$. The left figure shows the Langevin history of $\left\langle|\phi|^{2}\right\rangle$, using three different initial conditions but the same random number sequence in the stochastic process. Since $\mu=0.5$ is far from the critical value (and hence the minimal value of $A_{p}$ is well separated from zero), the memory of initial conditions is quickly erased. To have an indication for the size of the statistical fluctuations during the evolution, runs with a different random number sequence are shown in the figure on the right. Note that in a typical run the Langevin time $0<\theta<$ 250.
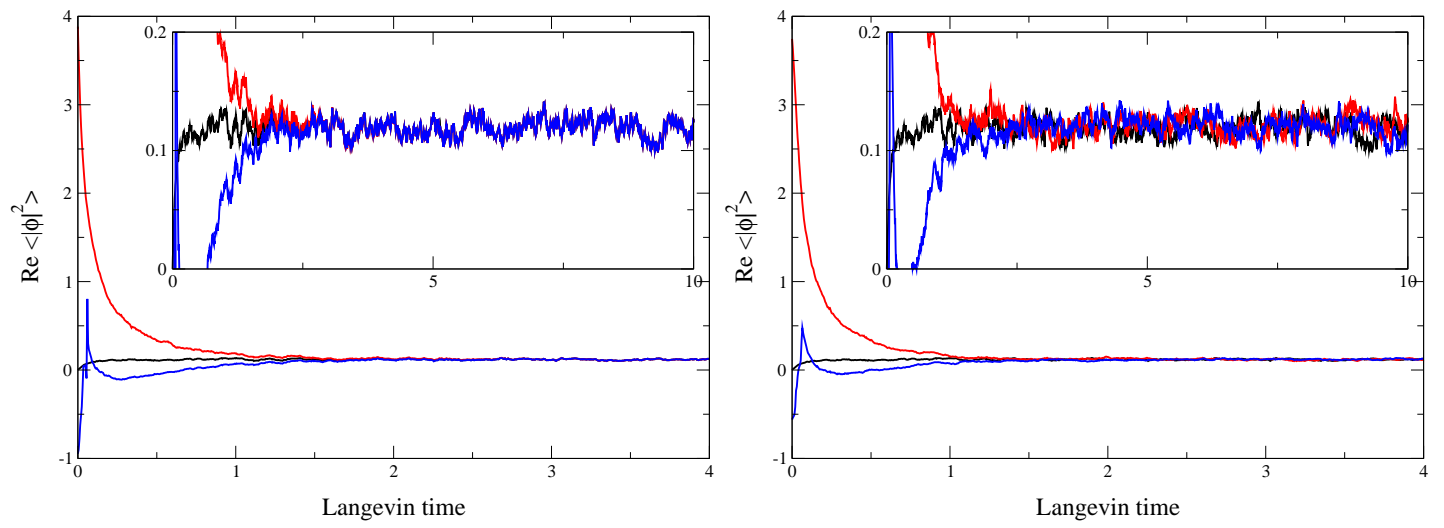

Figure 2: Independence of initial conditions: Langevin evolution of $\left\langle|\phi|^{2}\right\rangle$ with three different initial conditions using the same (left) and a different (right) random number sequence $\left(\mu=0.5, m=\lambda=1, N^{4}=4^{4}\right)$.

Expectation values are obtained by noise averaging and taking the limit $\theta \rightarrow \infty$. Provided again that $A_{p}>0$, we find from the analytical solution

$$
\begin{aligned}
& \lim _{\theta \rightarrow \infty}\left\langle\phi_{a,-p}^{\mathrm{R}}(\theta) \phi_{b, p^{\prime}}^{\mathrm{R}}(\theta)\right\rangle=\delta_{a b} \delta_{p p^{\prime}} \frac{1}{2 A_{p}} \frac{2 A_{p}^{2}+B_{p}^{2}}{A_{p}^{2}+B_{p}^{2}}, \\
& \lim _{\theta \rightarrow \infty}\left\langle\phi_{a,-p}^{\mathrm{I}}(\theta) \phi_{b, p^{\prime}}^{\mathrm{I}}(\theta)\right\rangle=\delta_{a b} \delta_{p p^{\prime}} \frac{1}{2 A_{p}} \frac{B_{p}^{2}}{A_{p}^{2}+B_{p}^{2}}, \\
& \lim _{\theta \rightarrow \infty}\left\langle\phi_{a,-p}^{\mathrm{R}}(\theta) \phi_{b, p^{\prime}}^{\mathrm{I}}(\theta)\right\rangle=\varepsilon_{a b} \delta_{p p^{\prime}} \frac{i}{2} \frac{B_{p}}{A_{p}^{2}+B_{p}^{2}} .
\end{aligned}
$$



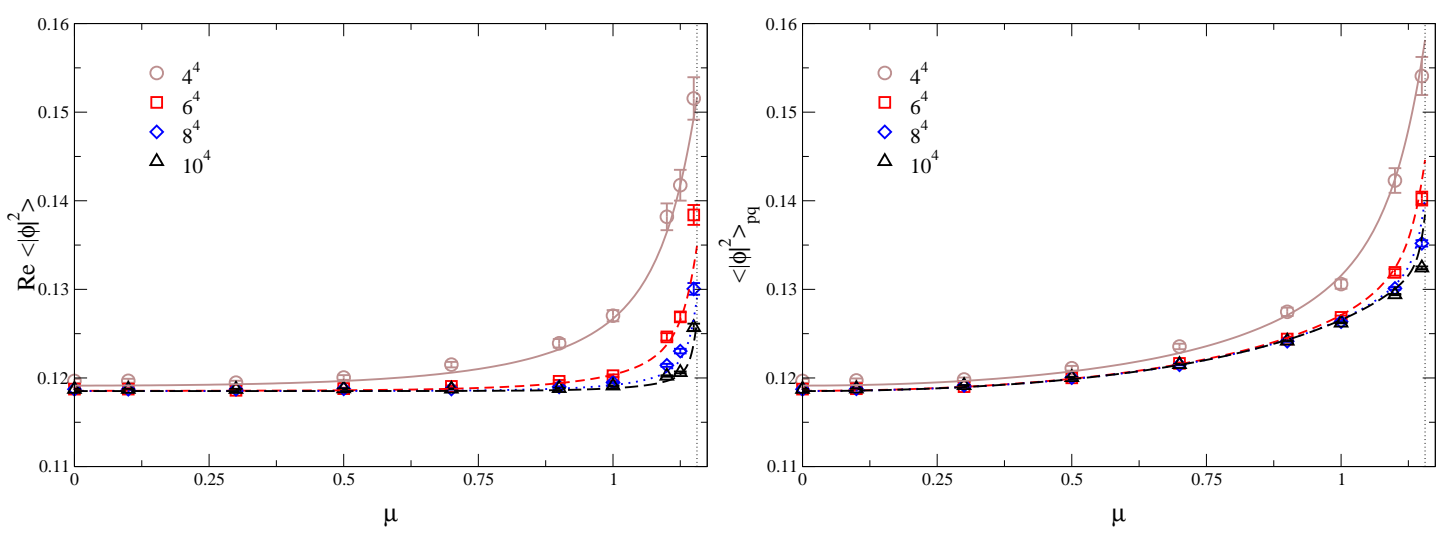

Figure 3: Comparison between the mean field predictions (lines) and the numerical data (symbols) for $\left\langle|\phi|^{2}\right\rangle$ in the full (left) and phase quenched (right) theories in the Silver Blaze region. The vertical dotted line indicates the mean field estimate for the critical chemical potential.

So far interactions have been neglected in this analytical calculation. An elegant way to include interactions on the mean field level is by looking for fixed points of the Langevin equations for the two-point functions above, using a Gaussian factorization [10]. After the dust settles, the result is a simple shift in the effective mass parameter, $m^{2} \rightarrow m^{2}+4 \lambda\left\langle|\phi|^{2}\right\rangle$, as expected. The critical value of the chemical potential in the mean field approximation then follows from $m^{2}+4 \lambda\left\langle|\phi|^{2}\right\rangle+2(1-$ $\cosh \mu)=0$, where $\left\langle|\phi|^{2}\right\rangle$ is determined by a self-consistent gap equation. For the parameters used here we find $\mu_{c}=1.15$, in agreement with the numerical estimate.

We can now compare the nonperturbative results from the numerical solution of the complex Langevin equations with the mean field estimates on a finite lattice. This is shown in Fig. 3 for $\left\langle|\phi|^{2}\right\rangle$ and in Fig. 4 for $\langle n\rangle$. Since at $\mu=0$ the action is real, the value of $\left\langle|\phi|^{2}\right\rangle$ at $\mu=0$ is obtained with real Langevin dynamics. This provides a nontrivial check of the numerical code. We observe excellent agreement between the simulations and the analytical results, including finitesize effects. This indicates that complex Langevin dynamics is successful, but also that the mean
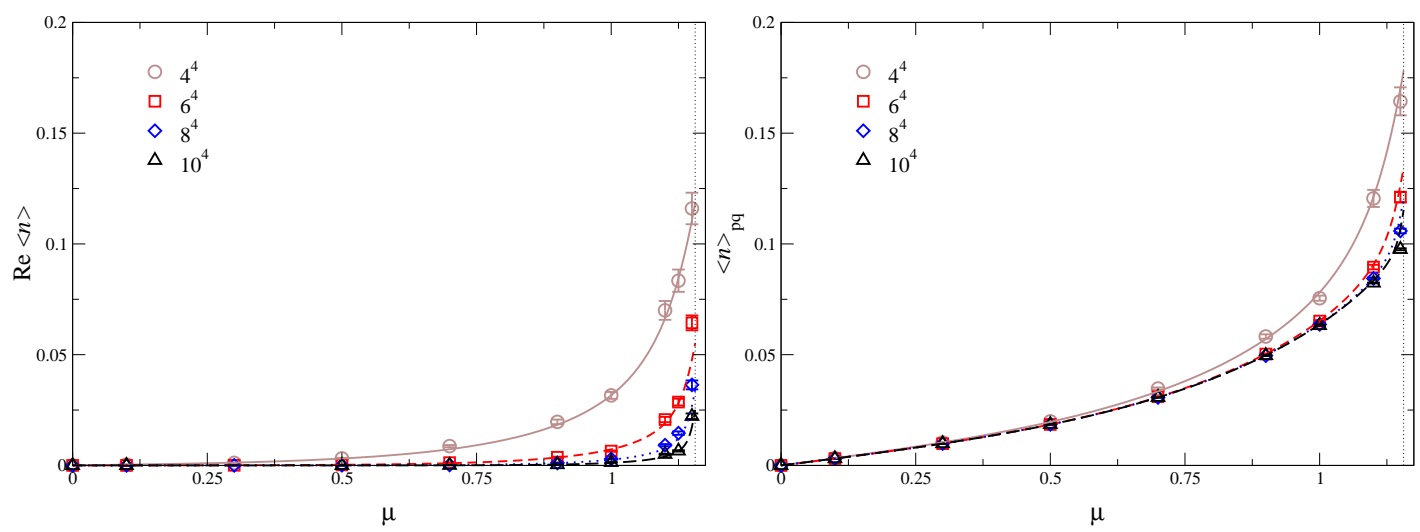

Figure 4: As above, for the density $\langle n\rangle$. 

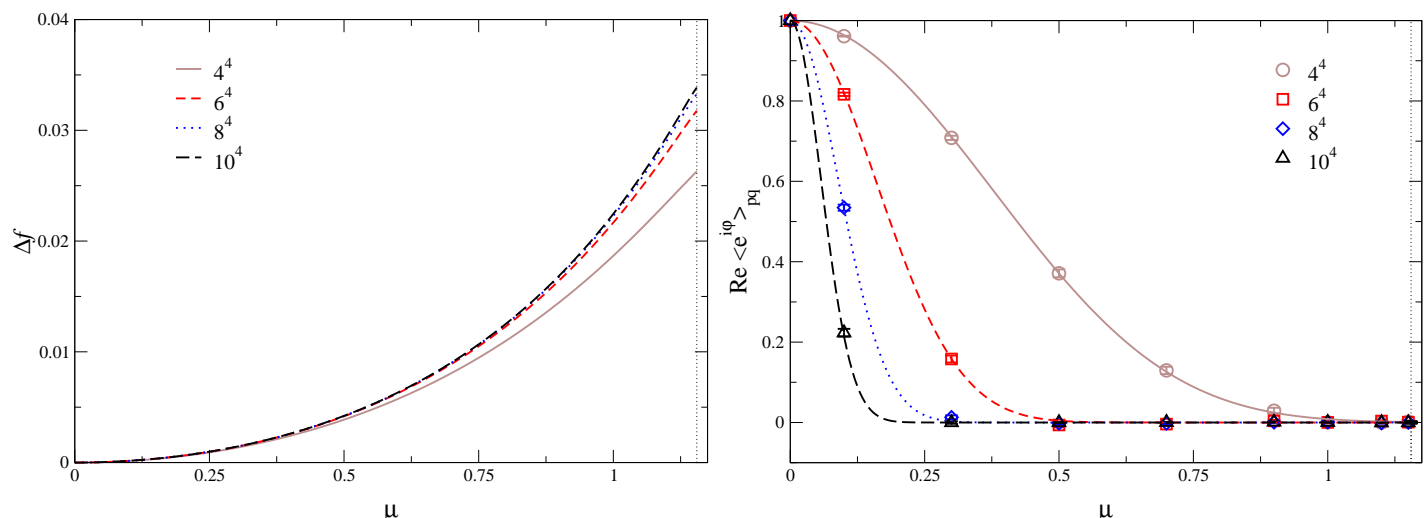

Figure 5: Left: difference $\Delta f$ between the free energy densities of the full and the phase quenched theories in the mean field approximation. Right: as in the preceding figures, for the average phase factor $\left\langle e^{i \varphi}\right\rangle_{\mathrm{pq}}$ in the phase quenched theory.

field approximation is adequate and the theory is effectively weakly coupled.

The results so far answer three of the questions raised in the Introduction. What about the severity of the sign problem? From the pronounced difference between the full and the phase quenched theory, one can expect the sign problem to be severe. This is quantified by studying the average phase factor,

$$
\left\langle e^{i \varphi}\right\rangle_{\mathrm{pq}}, \quad \quad e^{i \varphi}=\frac{e^{-S}}{\mid e^{-S \mid}},
$$

where the expectation value is taken in the phase quenched theory, with real weight $\left|e^{-S}\right|$. This phase factor is relevant for reweighting and is expected to vanish exponentially in the thermodynamic limit, since

$$
\left\langle e^{i \varphi}\right\rangle_{\mathrm{pq}}=\frac{Z_{\mathrm{full}}}{Z_{\mathrm{pq}}}=e^{-\Omega \Delta f}
$$

where $\Delta f$ is the difference between the free energy densities in the full and the phase quenched theories. The mean field results for the difference between the free energies are shown in Fig. 5 (left). We note a rapid convergence in the thermodynamic limit. Inserting these results for $\Delta f$ in the exponential $e^{-\Omega \Delta f}$ yields the average phase factor. In the numerical simulations the phase factor is measured directly. A comparison between the two is shown in Fig. 5 (right). We observe again excellent agreement. The severeness of the sign problem is manifest. As mentioned above, this is necessary and does not affect complex Langevin dynamics in practice.

\section{Outlook}

The question posed in the title and made more specific in the Introduction has been answered positively for the relativistic Bose gas at finite chemical potential considered here. In this model the sign problem is required to yield the correct physics, summarized by the Silver Blaze problem, and it is severe. Nevertheless, complex Langevin dynamics can be applied without encountering 
(numerical) problems affecting e.g. stability or convergence. The outcome of the numerical simulations can be understood from a comparison with mean field theory. Moreover, the inner workings of the algorithm can be analysed as well, relying essentially on the weakly coupled nature of this theory.

Does this mean that stochastic quantization can easily be applied to other theories with a complex action due to a nonvanishing chemical potential? We are currently studying complex Langevin dynamics in a variety of models, including the three-dimensional XY model at nonzero chemical potential, and extending our analysis of QCD with static quarks. At this stage it is fair to say that easily is slightly too optimistic.

\section{Acknowledgments}

Discussions with Philippe de Forcrand, Simon Hands, Frank James, Erhard Seiler, Kim Splittorff and Ion-Olimpiu Stamatescu are greatly appreciated. I am grateful to the Royal Society for Conference support.

\section{References}

[1] P. de Forcrand, PoS LAT2009 (2009) 010

[2] G. Parisi and Y. s. Wu, Sci. Sin. 24 (1981) 483.

[3] G. Parisi, Phys. Lett. B 131 (1983) 393.

[4] J. R. Klauder, Stochastic quantization, in: H. Mitter, C.B. Lang (Eds.), Recent Developments in High-Energy Physics, Springer-Verlag, Wien, 1983, p. 351.

[5] P. H. Damgaard and H. Hüffel, Phys. Rept. 152 (1987) 227.

[6] G. Aarts and I. O. Stamatescu, PoS LAT2008 (2008) 176 [0809.5227 [hep-lat]].

[7] G. Aarts and I. O. Stamatescu, JHEP 0809 (2008) 018 [0807.1597 [hep-lat]].

[8] T. D. Cohen, Phys. Rev. Lett. 91 (2003) 222001 [hep-ph/0307089].

[9] G. Aarts, Phys. Rev. Lett. 102 (2009) 131601 [0810.2089 [hep-lat]].

[10] G. Aarts, JHEP 0905 (2009) 052 [0902.4686 [hep-lat]]. 